\title{
Exploring accreditation from the front lines: A qualitative study of staff experiences \& perceptions of hospital accreditation
}

\author{
Katie N Dainty*1,2, Douglas Sinclair ${ }^{3}$, Cathy O'Neill ${ }^{3}$, Sherra Solway ${ }^{3}$, Bianca Seaton ${ }^{1}$ \\ ${ }^{1}$ North York General Hospital, Canada \\ ${ }^{2}$ Institute of Health Policy, Management and Evaluation, University of Toronto, Canada \\ ${ }^{3}$ St. Michael's Hospital, Canada
}

Received: April 5, 2019

DOI: $10.5430 /$ jha.v8n4p24
Accepted: April 28, 2019

Online Published: June 5, 2019

\begin{abstract}
Objective: Relative to the global investment in hospital accreditation programs overall, both the total amount and methodological quality of existing accreditation research is mediocre. To address this apparent gap in knowledge, we undertook a study of the experiences and perspectives of hospital staff, those most underrepresented in the accreditation research literature, including those working on the front lines and non-leaders who may or may not have been directly involved in the preparation for the accreditation survey visit.

Methods: Design: Qualitative descriptive interview study. Setting: Tertiary care teaching hospital in Toronto, Ontario Canada. Study Participants: Program directors, unit managers, physicians, nurses, health discipline professionals (HDP), and non-clinical staff.

Results: We have grouped what we heard from the data into three major groups of findings which we have titled: a) perceptions of the purpose of accreditation; b) the "work" of accreditation; and c) dissemination gaps regarding results. Informative finding from this study include the fact that participants put surprising value on the accreditation process, although mistaken feel it is mandatory; that the "work" of accreditation can be disappointing and feels disconnected from the bigger QI picture, and that the disconnect also exists in terms of their knowledge of and belief in the actual results of the process.

Conclusion: These findings point to potential gaps in the accreditation culture which can be detrimental to the impact of the accreditation process. Such gaps likely exist in other health care organizations and could inform similar evaluations in other settings.
\end{abstract}

Key Words: Hospital accreditation, Staff experience, Qualitative, Accreditation Canada

\section{INTRODUCTION}

Hospital accreditation has become a popular process for validating the quality, safety, and efficiency of healthcare organizations and is embedded within the healthcare systems of more than 70 different countries worldwide. ${ }^{[1]}$ Accred- itation is defined as "both a self-assessment and external peer assessment process used by health care organizations to accurately assess their level of performance in relation to established standards and to implement ways to continuously improve". ${ }^{[2]}$

\footnotetext{
*Correspondence: Katie N Dainty; Email: katie.dainty@utoronto.ca; Address: North York General Hospital, 4001 Leslie Street, Toronto, Ontario Canada.
} 
Accreditation Canada is an independent, not-for-profit organization that accredits health organizations in Canada. They have been accrediting hospitals since 1958 and currently accredit more than 7,000 sites in health care and social services across 5 continents. ${ }^{[3]}$ They use their Qmentum Accreditation Program to provide organizations with an independent, third-party assessment using standards built developed with best practices and subject matter experts. It is intended to involve all members of an organization, from the board of directors to frontline staff as well as members of the community including patients and families and community partners, and it focuses on areas such as how to make better use of resources, increase efficiency, enhance quality and safety, and reduce risk. ${ }^{[4]}$ An overall report is prepared which outlines how an organization scores against the National standards and a cumulative accreditation standing is given to each organization from "Not Accredited" to "Accredited with Exemplary Standing". [3]

Participation in a hospital accreditation survey involves significant investments of time, money, and human resources. Yet the benefits of this practice have been questioned and despite several recent systematic reviews, the evidence base supporting the efficacy of accreditation in improving patient outcomes and enhancing the quality and safety of clinical care is limited and thought to be incomplete. ${ }^{[5,6]}$ This is in part due to the inherent challenge of trying to assess a complex, heterogeneous intervention like "accreditation" and also because of the limitations of the few existing studies assessing the effects of accreditation on quality of care. ${ }^{[6,7]}$

Relative to the global investment in hospital accreditation programs overall, both the total amount and methodological quality of existing accreditation research is mediocre. ${ }^{[7]}$ As a result, there is much we do not understand about it, including about how hospital staff actually experience and engage with accreditation programs, as well as the impact of accreditation on the front lines of healthcare organizations. To address this apparent gap in knowledge, we undertook a study of the experiences and perspectives of hospital staff, including those working on the front lines and non-leaders who may or may not have been directly involved in the preparation for the accreditation survey visit.

\section{DESIGN}

\subsection{Study setting}

St. Michael's Hospital is a large tertiary care teaching hospital in Toronto, Ontario Canada with 455 beds, 6,100 employees and an average of 26,000 admissions and 527,000 ambulatory care visits per year. St. Michael's Hospital participates in Accreditation Canada's Qmentum accreditation program every four years as part of its ongoing quality improvement.
As part of this program, the organization undergoes a rigorous evaluation process including a self-assessment, standardized questionnaires to assess work culture, patient safety culture, patient experience, governance functioning and external peer surveyors conducting an on-site survey. In the last two accreditation cycles (2012 and 2016), St. Michael's Hospital has received "exemplary standing".

\subsection{Study design}

The study employed qualitative descriptive methodology. Qualitative research emphasizes understanding the different meanings, perceptions, and experiences that people have of social phenomena and processes and is particularly useful in unpacking some of the complex issues inherent to quality improvement, such as the ways in which interpersonal and workplace relations may be impacted ${ }^{[8]}$ which made it ideal for this study.

\subsection{Research sample and selection}

We used a combination of stakeholder and maximum variation sampling strategies in this study, and our overall sampling approach was purposive. Stakeholder sampling involves identifying the major participants who are involved in designing, giving, receiving, or administering the initiative being investigated, and who might be affected by it. ${ }^{[9]}$ The maximum variation sampling approach further ensured that we included individuals who cover the spectrum of positions, perspectives and opinions. ${ }^{[10]}$

We approached participants from multiple stakeholder groups to ensure there was representation from different components of the experience of Accreditation in our sample: program directors, unit managers, physicians, nurses, health discipline professionals, and non-clinical staff. We deliberately sought physicians and staff who did not necessarily lead or play an active role in preparing their unit and colleagues for the on-site survey visit or participate in the audit itself, as well as those who were more actively involved. We also aimed to include participants with varying years of experience in both their profession and as employees of St. Michael's Hospital Hospital, those who work different shifts, and those with different experiences interacting with Surveyors.

\subsection{Data collection}

The primary method for collecting data was in-depth qualitative interviews with the hospital staff. This format allows the interviewee to guide the conversation while at the same time providing direction around certain topics. The interviews were conducted by research team member (MBS), a trained interviewer with qualitative research expertise who was not involved in Accreditation or hospital operations in 
any way. The interview transcripts were supplemented with field notes.

To manage interview dynamics, minimize social desirability bias, and probe emergent findings in situ, all interviews were conducted in person (in private offices and meeting rooms at the hospital) or by telephone. The interviews ranged from 10-45 minutes in length and were all audio-taped and transcribed verbatim. Separate interview guides for the unit managers and directors, physicians, nurses/HDP and support staff members were developed by the research team and assessed for face and content validity via pilot testing before commencing the interviews.

\subsection{Data analysis and management}

In keeping with the iterative process of qualitative methodology, data analysis occurred in conjunction with data collection. The interview transcripts were reviewed and coded by the lead research team members (KND and MBS), who both have qualitative methodological expertise. Comparisons within and across interviews were conducted in order to continuously monitor emerging themes and general areas for further exploration. Despite the diversity in roles of the respondents, there was quite a bit of agreement among what the participants said, and we reached thematic saturation ${ }^{[13]}$ on several topics. The findings of the analysis were discussed with research colleagues on an ongoing basis.

The data was analyzed according to standard thematic analysis techniques. ${ }^{[11]}$ Descriptive codes were first attached to segments of the text in each transcript. The descriptive codes were then grouped into broad topic-oriented categories and all text segments belonging to the same category were compared. Ultimately the topic-oriented categories were further refined and formulated into fewer analytic categories through an inductive, iterative process. Data management was facilitated using NVivo software (version 11).

\section{Findings}

Over the course of 5 months (Oct 2016-March 2017) we interviewed 36 staff members and physicians from across the hospital. Our sample included individuals from nursing, medicine, the health discipline professions, administration, support staff, and various leadership positions, with an equally broad range from one to greater than forty years of experience working at the hospital. Interviews ranged from 10-45 minutes and were audio recorded and transcribed verbatim for analysis.

We have grouped what we heard from the data into three major groups of findings which we have titled: a) perceptions of the purpose of accreditation; b) the "work" of accreditation; and c) dissemination gaps regarding results. Exemplar quotes for each theme are provided in Table 1.

\subsection{Perceptions of the purpose of accreditation}

In order to uncover potential assumptions and inform areas for future knowledge translation related to quality and safety activities at St. Michael's Hospital Hospital, we directly asked participants about their understanding of the purpose and value of hospital accreditation. We heard very similar responses across the data set; hospital staff who participated in this research were generally of the opinion that accreditation is both a purposeful activity and one that is important for St. Michael's Hospital to undertake.

Participants commonly used the terms "accountable/accountability", and "reflect/reflection" when sharing their perspectives on the purpose and value of accreditation. As the quotations in Table 1 illustrate, accreditation activities were seen as helping to fulfill the hospital's duty to be answerable, internally and externally, for the care provided to patients, in part by creating an opportunity for critical examination and consideration of daily work practices at both the micro (individual) and macro (unit or institution) levels.

Of interesting note, all of the participants in this study held the belief that accreditation is a mandatory activity that the hospital must necessarily undertake or face severe sanctions. Several participants shared their assumptions that the hospital would lose funding from the Ontario Ministry of Health and Long-Term Care or otherwise be "shut down" if the Qmentum Accreditation survey processes were failed or declined. Although participants were uncertain about the veracity of these sanctions, the assumption that the hospital was required to engage in accreditation - and required to do well - was widespread in our sample, even though it is actually a completely voluntary exercise.

\subsection{The "Work" of accreditation}

While the participants in this study were almost unanimously in agreement that accreditation is an important activity for St. Michael's Hospital Hospital, there was no similar consensus about the extent, or the value of the work required to prepare for an on-site visit. The much wider continuum of how participants spoke about the work involved in accreditation reflects the diversity of roles held by participants in our sample. Many of the staff members we spoke to described themselves as "least involved" with preparing their units for accreditation or who reported their daily life at work was "minimally impacted" by the accreditation preparations. Where high levels of engagement and/or support of the process was demonstrated by these staff members it was often attributed to a particularly inspiring, enthusiastic, and organized local accreditation team lead.

ISSN 1927-6990 E-ISSN 1927-7008 
Table 1. Exemplar Interview Quotes

\begin{tabular}{|c|}
\hline Perceptions of the Purpose of Accreditation \\
\hline $\begin{array}{l}\text { "I think it's a really important process, for sure, just to make sure that we are getting in the right standards and that we can compare ourselves to other } \\
\text { hospitals and make sure that we're improving and aligned with the rest of the world." [EA-P14] }\end{array}$ \\
\hline $\begin{array}{l}\text { "Well, I think it's a good exercise for looking at things that we're doing well and areas that need improvement, and having some oversight and } \\
\text { accountability and transparency, that you have an outside agency coming in and seeing how we're practicing and providing safe care." [EA-P18] }\end{array}$ \\
\hline $\begin{array}{l}\text { “Oh, I think it's imperative and important. I think if we didn't participate any kind of accreditation process, we really wouldn't know if we're abiding by } \\
\text { certain standards across our hospital. I think, especially in today's day and age with information privacy and confidentiality, we want to make sure that } \\
\text { a certain set of standards are being met.” [EA-P13] }\end{array}$ \\
\hline The “Work" of Accreditation \\
\hline $\begin{array}{l}\text { "It was a lot of preparation for a little bit of lunch bag letdown...It was just, we kind of had a bit of a laugh at it, because there's so much work that goes } \\
\text { into preparing, and you didn't even really have a chance to blow your horn and say how well we've done with our program." [EA-P18] }\end{array}$ \\
\hline $\begin{array}{l}\text { "It is kind of like a black box, even when accreditation came, I didn't really know what happened or what they were doing. I didn't see the accreditors } \\
\text { walk by. But it would have been really interesting to see what they were actually assessing and how they were assessing it.” [EA-P14] }\end{array}$ \\
\hline $\begin{array}{l}\text { "It was like, hey, they were supposed to be [on other unit] only for an hour. What about us? We want our turn... We were ready and eager to show } \\
\text { off." [EA-P16] }\end{array}$ \\
\hline $\begin{array}{l}\text { "I feel that we rush, and we prepare for the accreditation, doing everything that we should have been doing from day one, just for the week or the few } \\
\text { days that the accreditors are in the hospital, and specifically in the unit. So, I feel that there is a lot of pressure put on everyone, including the nurses, } \\
\text { the CAs, everyone, to be tip-top form. Policies are reviewed. Certain standards are changed dramatically, because that's not the way that they should } \\
\text { have been done... but this should be happening on a continual basis" [EA-P12] }\end{array}$ \\
\hline $\begin{array}{l}\text { "Accreditation right now as it stands is one big test. And once the test is over, everyone kind of breathes again and says, okay, now we can go back to } \\
\text { doing what we have always done, which is maybe it's eating in your office or whatever." [EA-P5] }\end{array}$ \\
\hline "Whatever is required for accreditation, we should not have it as a separate project, but consistently review and monitor those things." [EA-P24] \\
\hline "So I see it as a big continuum and not just like, here we go for the next two months, everybody wear a clean shirt kind of thing." [EA-P32] \\
\hline $\begin{array}{l}\text { “Okay it's over. Everyone go back to normal”. That's what it was like. It sounds horrible. But it was heightened tension. Everything's perfect. } \\
\text { Probably, the unit's been the cleanest it's ever been for the whole year that week. People constantly running around saying, “That shouldn't be there. } \\
\text { That shouldn't be there.” And then, after, it was like, “Oh, okay, it's over,”... like a weight lifted off. [EA-P12] }\end{array}$ \\
\hline $\begin{array}{l}\text { “There's such a huge burden put on us once every what, four years or three years or whatever it is to learn all this stuff, and then it goes away. It's a lot } \\
\text { of time, I think, and money spent on things that, I don't know, does it matter? Maybe it does. Maybe we learn a lot in those couple of weeks, the } \\
\text { pre-lead to accreditation, and we retain it... But I don't retain it personally. It's gone... So for a couple of days, nice people will come and ask you } \\
\text { questions, and then it's over. And then we go back to doing what we do, caring for people at the hospital.” [EA-P9] }\end{array}$ \\
\hline $\begin{array}{l}\text { "People, I think, bought into the intention, but I think they find it a lot of work and not sure that it was the most efficient process in the world and } \\
\text { whether or not we could have achieved similar outcomes with less work." [EA-P35] }\end{array}$ \\
\hline Dissemination Gaps Regarding Results (“Exemplary Standing? I had no idea”) \\
\hline $\begin{array}{l}\text { "I think we just got an email. If I remember correctly, I think we did fairly well. I can't recall what the items were that we were flagged on, if I need to } \\
\text { be honest." [EA-P13] }\end{array}$ \\
\hline $\begin{array}{l}\text { "I forget what it was but it was in the high nineties, like 98, } 99 \text { percent compliant with the standards. You know, it's kind of like oh that's great, I'm } \\
\text { very happy we did very well. But at the same time, maybe it's a missed opportunity for us to get some really good, meaningful feedback on how we } \\
\text { can get better as an organization. Most hospitals seem to do very well, when we know -- and again, I don't want to sound like I'm overly negative or } \\
\text { critical -- that there are lots of opportunities to improve.” [EA-P28] }\end{array}$ \\
\hline
\end{tabular}

Nevertheless, many participants characterized the work as "a lot of preparation for little interaction". After months of team meetings, document reviews, unit projects, policy updating, individual learning, and a "flurry of emails" to prepare for the on-site visit, the minimal (if any) interaction that most participants had with the Surveyors, the exclusion of their unit from direct observation by the Surveyors, and a general lack of clarity about what the Surveyors were doing when on-site, was experienced as a letdown that left participants feeling disappointed and questioning the value of all of the work.

In addition, several staff members described their managers and/or colleagues as nervous about the on-site visit and reluctant to interact with surveyors. We heard from a few participants that some of their colleagues purposefully book the week of the on-site visit off work or arrange their work schedules to avoid having to speak with and be observed by the surveyors, lest they say (answer a question) or do something wrong and make their unit or the hospital look bad.

Those who were interviewed and observed by the Surveyors during the onsite Accreditation Survey expressed that the visitors were very pleasant, professional, and complimentary, but shared mixed reactions to the casual nature of the conversations and the overall brevity of the interaction. Some told us they enjoyed the relaxed interaction and appreciated having the opportunity to speak briefly about their jobs with an interested visitor. Others expressed surprise, telling us they 
had diligently prepared themselves (and been prepared by the unit leaders) to be "tested". This misalignment between expectations and experiences was somewhat confusing. In addition, many reflected that there is a severe lack of continuity of review and reinforcement outside of the Accreditation period. Many participants suggested that the exercise would be "more real" and "more valuable" to hospital staff if the accreditation preparation activities took place on an ongoing basis, rather than sporadically every few years.

\subsection{Dissemination gaps regarding results}

We were surprised to find that the majority of the participants were actually unaware of the results of the Accreditation exercise. Several commented that they "likely received an email" but could not speak to what the outcome was despite their involvement in the process. When we shared the fact that St. Michael's Hospital had received Exemplary Standing and scored almost $100 \%$ on all standards, the news was received by many participants as a source of pride and validation. However, others received this information with skepticism about the validity of the scoring mechanism and questioned the context of these results.

\section{Discussion}

This study produced a unique and robust dataset of the frontline staff and physician perspectives on the process of hospital Accreditation. Common themes throughout the data included unpacking participants understanding of the purpose and value of the accreditation process, their impressions of the "work" of accreditation, and the impact of dissemination gaps regarding the outcome of the process.

Accreditation was unanimously viewed by participants as an activity that supports the quality and safety goals of the hospital and helps fulfill the hospital's mandate to be internally and externally accountable for the care provided. It is seen as an exercise that is helpful to the hospital in achieving the goal of providing excellent patient care by highlighting to individual employees/units their areas of strength and opportunities for improvement. However, it is not seen as a voluntary activity for the hospital, per se, but rather assumed to be something that all hospitals are is required to undertake. The assumption that accreditation is somehow related to continuing hospital operations and/or funding was common among the interview participants. This may contribute to why we heard from some participants that accreditation can be a rather anxiety-inducing activity.

Preparing for the hospital Accreditation process is a massive undertaking which requires a phenomenal amount of coordination and communication. Some of the findings reported here can lead to improvements in the process for frontline staff and others are a consequence of the nature of how the Surveyors choose to review each institution. What was most compelling was the very solid grasp the participants had on why Accreditation is important. Stemming from that, the participants were able to quite clearly articulate why they felt that the preparatory work should be a routine set of activities conducted on an ongoing basis. This likely supports the theory on which successful quality improvement is based - the concept of an improvement culture that is continuously reviewing and revising $;{ }^{[12]}$ always being ready versus having to prepare for an event or a review.

Finally, a result of "exemplary standing" seems to actually be a common Accreditation result in hospitals in the same geographic area as our study hospital and so we were interested to see participants' perspectives on the meaning of these results. While it is obviously excellent for an organization to receive the highest commendation, the translation of these results to staff has not previously been explored. In the case of our study sample, the apparent lack of knowledge or interest in the results of such an intensive process begs the question of whether there has actually been a measurable impact on the organization from participating in the Qmentum Accreditation Program or if staff just see it as a siloed exercise. More importantly, when the results were shared there was some definite skepticism about the validity of the outcome, i.e. "receiving 100\%" or "exemplary standing", given what staff know to be true in the day-to-day context of their lives at work.

Accreditation in general tends to generate strong, mixed reactions from the healthcare professionals involved in this practice, with some endorsing it and others highly critical. ${ }^{[5]}$ Previous research investigating the attitudes, perceptions, and experiences of healthcare professionals with hospital accreditation have produced mixed results. As is the case in our study, in general many seem to support accreditation, ${ }^{[13]}$ view it as an effective method of promoting high quality organizational processes and patient safety, ${ }^{[7]}$ and value participating in the process. ${ }^{[14]}$ However, concerns about the financial cost, human resource investment, and bureaucratic process are common, as are questions about whether accreditation has a significant impact on the quality of healthcare services. ${ }^{[15]}$ One study documented the unintended negative effects of hospital accreditation on the learning environment of medical students and trainees, including decreased clinical learning opportunities, increased non-clinical workload, and violation of professional integrity during the accreditation process. ${ }^{[16]}$

The results from this work represent the one of very few of the front-line perspective on the process of accreditation. 
These insights obviously provide useful direction for improvement in local process but more importantly, at a higher level they prompt us to think differently about the philosophy of the process of accreditation itself. Is Accreditation as it is currently organized contributing to a continuous culture of best practice and quality? Does the hospital's standing (i.e., exemplary or otherwise) hold any meaning to those frontline staff and clinicians holding down the fort day in and day out? Accreditation is a quality assessment exercise that has become standard in hospitals across the globe and is a key component of the quality and patient safety agenda of many health care organizations. However, when any initiative becomes commonplace, assumptions begin to enter into our collective understanding of what the initiative means, why we do it, and why it is important.

\section{Strengths and limitations}

As with all research, this study has strengths and limitations. We feel this is a robust sample of in-depth interview data and that the data collection and analysis was conducted rigorously according to standard qualitative methods. Purposive sampling also allowed us to speak to a variety of stakeholders, therefore enriching the data. The sample is from a single site; however, we feel that while gathering data from multiple sites may have made the data broader it would not have been nearly as deep and therefore less useful. We do acknowledge that the unique experience of accreditation at the study hospital may have heavily influenced the themes which emerged and therefore would encourage more of this type of research in other organizations to look for similarities or incongruence in order to further our understanding.

\section{Conclusions}

This study produced a unique and robust dataset of the frontline staff perspective on hospital Accreditation. Informative findings from this study include the fact that participants put surprising value on the accreditation process, although mistaken feel it is mandatory; that the "work" of accreditation can be disappointing and feels disconnected from the bigger QI picture, and that the disconnect also exists in terms of their knowledge of and belief in the actual results of the process. These findings point to potential gaps in the accreditation culture which likely exist in other organizations and should similar evaluations in other settings.

\section{CONFlicts OF INTEREST Disclosure}

The authors declare they have no conflicts of interest.

\section{REFERENCES}

[1] Greenfield D, Braithwaite J. Developing the evidence base for accreditation of healthcare organisations: a call for transparency and innovation. Qual Saf Health Care. 2009; 18(3): 162-3. PMid:19467994. https://doi.org/10.1136/qshc. 2009.032359

[2] Jovanovic B. Hospital accreditation as method for assessing quality in health care. Arch Oncol. 2005; 13(3/4): 156.

[3] Accreditation Canada Website. Available from: https://accred itation.ca/accreditation/ - last accessed February 212019

[4] Health Standards Organization Website - Available from: https://healthstandards .org/assessment-programs/de velop-build-accreditation/ - last accessed February 21, 2019.

[5] Greenfield D, Braithwaite J. Health sector accreditation research: a systematic review. Intl J Qual Health Care. 2008; 20(3): 172-183. PMid:18339666. https://doi.org/10.1093/intqhc/mzn005

[6] Brubakk K, Vist GE, Bukholm G, et al. A systematic review of hospital accreditation: the challenges of measuring complex intervention effects. BMC Health Services Research. 2015; 15: 28090. PMid:26202068. https://doi.org/10.1186/s12913-015 $-0933-\mathrm{x}$

[7] Hinchcliff R, Greenfield D, Moldovan M, et al. Narrative synthesis of health service accreditation literature. BMJ Qual Saf. 2012; 21: 979-991. PMid:23038406. https://doi .org/10.1136/bmjqs-2 012-000852

[8] Pope C, Van Royen P, Baker R. Qualitative methods in research on healthcare quality. Qual Saf Health Care. 2002; 11: 148-152. PMid:12448807. https://doi.org/10.1136/qhc.11.2.148
[9] Morse JM. Chapter 8: Strategies for Sampling in Qualitative Nursing Research: A Comtemporary Dialogue. Eds Morse JM. Sage Productions, Newbury Park; 1991.

[10] Higginbottom G. Sampling Issues in Qualitative Research. Nurse Researcher. 2004; 12(1): 7.

[11] Boyatis R. Transforming Qualitative Information: Thematic Analysis and Code Development. Sage Publications, Thousand Oaks; 1998.

[12] Davies HTO, Nutley SM, Mannion R. Organizational culture and the quality of healthcare. Quality in Health Care. 2000; 9: 111-119. PMid:11067249. https://doi.org/10.1136/qhc.9.2.111

[13] Alkhenizan A, Shaw C. The attitude of health care professionals towards accreditation: A systematic review of the literature. J Family and Community Medicine. 2012; 19(2): 74-80. PMid:22870409. https://doi.org/10.4103/2230-8229.98281

[14] Greenfield D, Pawsey M, Braithwaite J. What motivates professionals to engage in the accreditation of healthcare organizations? Int J Qual Health Care. 2011; 23(1): 8-14. PMid:21084322. https: //doi.org/10.1093/intqhc/mzq069

[15] Davis MV, Cannon MM, Stone DO, et al. Informing the national public health accreditation movement: lessons from North Carolina's accredited local health departments. AJPH. 2011; 101: 1543-8. PMid:21778472. https://doi.org/10.2105/AJPH . 2011. 3001 99

[16] Ho MJ, Chang HH, Chiu YT, et al. Effects of hospital accreditation on medical students: a national qualitative study in Taiwan. Acad Med. 2014; 89: 1533-1539. PMid:25250745. https: //doi.org/10.1097/ACM.0000000000000481 